Equation of State, Occupation Probabilities and Conductivities in the Average Atom Purgatorio Code

P.A. Sterne, S.B.Hansen, B.G. Wilson, W.A. Isaacs

January 12, 2007

Radiative Properties of Hot Dense Matter Albufeira, Portugal

September 11, 2006 through September 15, 2006 
This document was prepared as an account of work sponsored by an agency of the United States Government. Neither the United States Government nor the University of California nor any of their employees, makes any warranty, express or implied, or assumes any legal liability or responsibility for the accuracy, completeness, or usefulness of any information, apparatus, product, or process disclosed, or represents that its use would not infringe privately owned rights. Reference herein to any specific commercial product, process, or service by trade name, trademark, manufacturer, or otherwise, does not necessarily constitute or imply its endorsement, recommendation, or favoring by the United States Government or the University of California. The views and opinions of authors expressed herein do not necessarily state or reflect those of the United States Government or the University of California, and shall not be used for advertising or product endorsement purposes. 


\title{
Equation of State, Occupation Probabilities and Conductivities in the Average Atom Purgatorio Code
}

\author{
P.A. Sterne ${ }^{a, *}$ S.B. Hansen ${ }^{a}$ B.G. Wilson ${ }^{a}$ W.A. Isaacs ${ }^{b}$ \\ ${ }^{a}$ Lawrence Livermore National Laboratory, P.O. Box 808, L-045,Livermore, CA \\ 94550 \\ ${ }^{\mathrm{b}}$ Lawrence Berkeley Laboratory, One Cyclotron Road MS-71R0259, Berkeley, CA \\ 94720
}

\begin{abstract}
We report on recent developments with the Purgatorio code, a new implementation of Liberman's Inferno model. This fully relativistic average atom code uses phase shift tracking and an efficient refinement scheme to provide an accurate description of continuum states. The resulting equations of state accurately represent the atomic shell-related features which are absent in Thomas-Fermi-based approaches. We discuss various representations of the exchange potential and some of the ambiguities in the choice of the effective charge $Z^{*}$ in average atom models, both of which affect predictions of electrical conductivities and radiative properties.
\end{abstract}

Key words: Equation of state, transport

\section{Introduction}

Accurate modeling of astrophysical and laboratory plasmas requires accurate equation of state (EOS) data for a variety of elements over a wide range of material conditions, from the solid state to the rare plasma and high energydensity regimes. However, precise EOS measurements exist only for a restricted set of materials at select temperature-density points. Equation of state models which give reliable data, constrained by experiments, are needed to fill in the gaps between data points. Ideally, such models should be valid for all elements and apply over a wide temperature and density range.

\footnotetext{
* Corresponding author: sterne1@llnl.gov
} 
Equation of state models typically consist of three parts: the cold curve, the ion-thermal contribution and the electron-thermal contribution. The Purgatorio code [1] is a recent implementation of Liberman's Inferno model [2], which belongs to a family of historically successful ion-in-cell or neutral-pseudo-atom (NPA) approaches to the electron-thermal contribution to the EOS [see, for example, Refs. [3-6]]. These models represent a significant advance in complexity beyond the commonly used Thomas-Fermi and related approaches [7-10].

We will briefly describe the Purgatorio code and its application to EOS and transport calculations. We also discuss some of the uncertainties associated with the model and its application, including the effects of changing the exchange and correlation potential and using various definitions of the average ion charge in transport calculations.

\section{The Purgatorio model}

Purgatorio numerically solves the Dirac equation for the major and minor components of the wave functions normalized to unity over all space, with analytic forms employed outside the Wigner-Seitz cell radius, $R_{W S}$, where the potential is set to zero. Continuum wave functions are computed on an adaptive energy grid to resolve detailed information on the density of states. Matching the numerical wave functions to analytic forms at $R_{W S}$ gives phase shifts $\delta_{\kappa}$, which are used in conductivity calculations. The energy grid, initially logarithmic, is refined to capture resonances which form in the continuum when bound states are destroyed by pressure ionization. Capturing the resonances is essential for a self-consistent calculation and is ensured either by the refinement described in Ref. [1] or by tracking changes in the phase shifts [6].

The bound and continuum electron densities are determined by populating the wave functions according to their statistical weights, modulated by the Fermi distribution function $f(\epsilon, \mu)=\left[1+e^{(\epsilon-\mu) / k T}\right]^{-1}$, with the chemical potential $\mu$ varied to ensure charge neutrality within $R_{W S}$ :

$$
\int_{0}^{R_{W S}} 4 \pi r^{2} \rho_{t o t}(r) d r=Z
$$

with

$$
\begin{aligned}
& \rho_{\text {tot }}(r)=\rho_{\text {bound }}(r)+\rho_{\text {continuum }}(r) \\
& 4 \pi r^{2} \rho_{\text {bound }}(r)=\sum_{i} f\left(\epsilon_{i}, \mu\right) 2\left|\kappa_{i}\right|\left\{P_{i}^{2}(r)+Q_{i}^{2}(r)\right\}
\end{aligned}
$$




$$
4 \pi r^{2} \rho_{\text {continuum }}(r)=\int_{0}^{\infty} d \epsilon f(\epsilon, \mu) \sum_{\kappa} 2|\kappa|\left\{P_{\kappa, \epsilon}^{2}(r)+Q_{\kappa, \epsilon}^{2}(r)\right\}
$$

where $P$ and $Q$ are the major and minor components of the wave function respectively.

The potential is determined from the total electron density and iterated to self-consistency using a Local Density Approximation (LDA) to the exchange and correlation potential [11].

Converged values of the electron density and its bound and continuum components are illustrated in Fig. 1(a) for $\mathrm{Cu}$ at solid density and a temperature of 1 $\mathrm{eV}$. The bound electrons have a clear shell structure, as labeled on the figure, which is absent in the Thomas-Fermi description (gray lines). The continuum electron distribution, which in the Thomas-Fermi approximation would be a smooth increasing function of $r$, shows a pronounced feature associated with the unbound $3 d$ state. Figure 1(b) shows the density-of-state like quantity $X(\epsilon)$ for this case, where the bound states are represented by delta functions at their negative-energy eigenvalues and the continuum $X(\epsilon)$ is given by

$$
X(\epsilon)=\int_{0}^{R_{W S}} d r \sum_{\kappa} 2|\kappa|\left\{P_{\kappa, \epsilon}^{2}(r)+Q_{\kappa, \epsilon}^{2}(r)\right\}
$$

In solid-density $\mathrm{Cu}$, the $3 d$ state is pressure ionized and appears as a resonance in the continuum, causing a significant deviation from the equivalent quantity for free waves $X^{\text {ideal }}(\epsilon)=p\left(1+\alpha^{2} \epsilon\right) /\left(\pi^{2} n\right)$, where $n$ is the ion density.

Previous calculations [1] used an adaptive energy grid method based on Gaussian quadrature to resolve detailed resonance structure in the density of states. We have now augmented this with a phase-shift-tracking method to ensure that all such resonance features are accurately represented. Figure 2 illustrates the ability of this combined approach to resolve resonance features in the density of states. This leads to greatly improved stability in energy differences between adjacent density-temperature points, resulting in significant improvements in the smoothness and consistency of the EOS.

\section{Equation of state data}

The accurate treatment of continuum electrons and the stringent numerical tolerances in Purgatorio permit the extraction of thermodynamic quantities, 
such as free energy, pressure and entropy, either directly or by numerical differentiation. Prototypical results from Purgatorio, including the principal Hugoniots of $\mathrm{Be}$ and $\mathrm{Al}$ and pressure vs. energy isochores for low-density $\mathrm{Al}$, have been published recently [1].

The EOS quantities are susceptible to changes in the choice of exchange and correlation potentials. This is illustrated in Fig. 3, in which Hugoniot curves are compared for the Thomas-Fermi-based QEOS model [10], Purgatorio using Hedin-Lundqvist (HL) LDA form for exchange and correlation, and Purgatorio with a Gupta-Rajagopal temperature-dependent exchange potential [12]. A simple ideal-gas ion-thermal contribution has been added to the Purgatorio results; this accounts for the differences between the HL results and QEOS at low pressures where ion-thermal contributions dominate the EOS. At higher pressures, where the temperatures are correspondingly higher, the electronthermal contribution to the EOS becomes dominant. The differences in the curves are then primarily due to differences in the treatment of the electron thermal components. In region of maximum compressibility on the Hugoniot, around 300 Mbar, the Purgatorio results indicate a more compressible EOS than QEOS. The temperature-dependent exchange term predicts a significantly more compressible Hugoniot than either the QEOS or the HL-based Purgatorio calculation, demonstrating the potentially strong sensitivity to the choice of exchange-correlation in these calculations.

The choice of exchange-correlation potential can also affect related quantities, such as density and temperature at which bound states become resonant in the calculation, thereby affecting the electron occupation probabilities. For example, in the Be Hugoniot calculations, the temperature-dependent exchange potential pushes the energy at which the 1s state becomes resonant to higher density at a given temperature, resulting in a more compressible Hugoniot. Such sensitivity to the exact location of ionization features can affect other quantities, such as electrical conductivities, as we will see in the next section.

Another point of uncertainty is the average ion charge $Z^{*}$, which, unlike other thermodynamic quantities, does not have a unique thermodynamically consistent definition. The most straightforward definition sets $Z^{*}$ to be the total number of continuum electrons:

$$
Z_{\text {continuum }}=\int_{0}^{R_{W S}} 4 \pi r^{2} \rho_{\text {continuum }} d r=\int_{0}^{\infty} f(\epsilon, \mu) X(\epsilon) d \epsilon
$$

This definition includes both the electrons in the ideal density of states, which have wave functions distributed throughout the material, and the quasi-bound electrons in the resonance features, whose wave functions have extensive tails but are fairly localized about the ion center (see Fig. 1). An alternative def- 
inition counts only the free electrons in the ideal density of states, excluding the population of quasi-bound or resonant states:

$$
Z_{\text {background }}=\int_{0}^{\infty} f(\epsilon, \mu) X^{\text {ideal }}(\epsilon) d \epsilon
$$

Finally, we can define the free electrons to be those on the surface of the Wigner-Seitz sphere:

$$
Z_{W S}=4 \pi R_{W S}^{2} \rho_{t o t}\left(R_{W S}\right) / n
$$

This definition largely excludes electrons in continuum resonances but can include a portion of any negative-energy bound states that leak out of the ion sphere. The electrons on the surface of the ion sphere are free to move between ions and can thus be considered extensive.

In most cases, $Z_{W S}$ and $Z_{\text {background }}$ are close to each other, and all three values tend to converge at high temperature and low density. However, at densities high enough to pressure ionize valence orbitals and temperatures low enough to significantly populate resonance states, the three quantities can differ widely: for example, in the case shown in Fig. $1, Z_{\text {continuum }}=11, Z_{W S}=2.21$, and $Z_{\text {background }}=1.65$.

A comparison of the three definitions of the free electron density with experimental data obtained from x-ray scattering of Be targets [13] is given in Fig. 4. The low-temperature points agree best with $Z_{\text {continuum, }}$, while the high temperature point agrees with the calculations excluding continuum resonances. These results are similar to those in Fig. 6 of Ref. [5], which shows SCAALP calculations that fall very near the Purgatorio $Z_{\text {continuum }}$ values, along with ACTEX [4] and NPA [3] calculations that broadly agree with the experimental data across the given temperature range.

\section{Transport quantities}

With knowledge of the self-consistent potential and the scattering phase shifts $\delta_{\kappa}$, we can construct the differential scattering cross sections. From these, we can calculate collision times, electrical resistivities [14] and other transport quantities through the extended Ziman formulation [15] following previous implementations in average-atom and NPA models [3,16-18]. The availability of the free wave functions should also enable us to calculate dynamic conductivities [21], which are closely related to opacities. 
The extended Ziman formulation for the electrical resistivity of liquid metals is based on linear response theory, where free electrons in a metal are uniformly accelerated until they collide with an ion and are scattered. The expression for the resistivity takes on a Drude-like form:

$$
\eta=\frac{1}{n Z_{i}}\left\langle\frac{1}{\tau}\right\rangle
$$

with $n Z_{i}$ is the charge carrier density and $\langle 1 / \tau\rangle$ an average electron-ion collision time which depends on the scattering cross section $\sigma(\epsilon)$ and the velocity distribution of the free electrons:

$$
\left\langle\frac{1}{\tau}\right\rangle=\frac{1}{3 \pi Z_{0}} \int_{0}^{\infty} \sigma(\epsilon) \frac{\partial f(\epsilon, \mu)}{\partial \epsilon} d \epsilon
$$

While $Z_{0}$, the number of free electrons per atom, is constrained on variational grounds to be identical to $Z_{\text {background }}$ when the Boltzmann equation is valid [16], there is no similar constraint on the number of charge carriers $Z_{i}$. We will therefore consider all three possible $Z_{i}$ values identified in the previous section in our conductivity calculations.

The scattering cross section is computed by integrating the differential cross section $d \sigma(p, \theta) / d \theta$, determined in the t-matrix formulation [15], over all possible scattering angles $\theta$ :

$$
\sigma(\epsilon)=\int_{0}^{\infty} q^{3} \frac{d \sigma(p, \theta)}{d \theta} S(q) d q
$$

with

$$
\frac{d \sigma(p, \theta)}{d \theta}=\frac{1}{p^{2}}\left(\left|\sum_{\kappa}\right| \kappa\left|e^{i \delta_{\kappa}(p)} \sin \left[\delta_{\kappa}(p)\right] P_{\ell}(\cos \theta)\right|^{2}+\left|\sum_{\kappa} \frac{|\kappa|}{i \kappa} e^{i \delta_{\kappa}(p)} \sin \left[\delta_{\kappa}(p)\right] P_{\ell}^{1}(\cos \theta)\right|^{2}\right)
$$

where $\ell=\kappa$ for $\ell>0$ and $\ell=-\kappa-1$ for $\ell<0, P_{\ell}$ and $P_{\ell}^{1}$ are the Legendre and associated Legendre polynomials, respectively, the relativistic dispersion relation $p^{2}=\epsilon\left(2+\epsilon \alpha^{2}\right)$ is assumed, and the integration is performed over the momentum transfer vector $q^{2}=2 p^{2}(1-\cos \theta)$. In order to obtain the proper behavior of calculated resistivities in the strong coupling regime, we integrate Eq. (11) using only the inelastic portion of the structure factor $S(q)$, following Refs.[19,20]. A complete description of the dependence on $S(q)$ is given in Ref. $[14]$. 
Calculated resistivities for $\mathrm{Al}$ and $\mathrm{Cu}$ using the three possible $Z_{i}$ are given in Fig. 5 along with experimental values obtained by DeSilva and Katsouros in wire vaporization experiments [22]. At the lowest densities, the three values are nearly equal and the calculated resistivities agree well with the data. But as the density increases, the pressure ionization of valence orbitals leads to resonance structure in the continuum, causing significant variation in the predicted density at which the metal-non-metal transition occurs with its characteristic sharp drop in resistivity. The variations are larger in $\mathrm{Cu}$, with 11 electrons in its $3 d$ resonance, than for $\mathrm{Al}$, with 3 electrons in $3 p$.

The large differences between the three definitions of $Z^{*}$ in the solid state and warm dense matter conditions can help to establish the proper choice of $Z^{*}$ for distributed transport quantities. This may not be the same as the $Z^{*}$ determined by microscopic probes such as the x-ray scattering data shown in Fig. 4. In fact, comparisons with measured conductivities of transition metals at standard conditions suggest that $Z_{\text {continuum }}$ is the least reliable choice for the number of charge carriers; experiments in the warm dense matter regime also support this conclusion [23]. Finally, we note that the predicted density of the metal-non-metal transition is also quite sensitive to the choice of exchange and correlation potential because of the strong influence of this potential on the electronic structure.

\section{Conclusions}

We have described the code Purgatorio, a new implementation of the Inferno model with a highly accurate treatment of continuum wave functions and stringent numerical tolerances. Purgatorio can provide the electron-thermal component of equation of state data for use in hydrodynamic modeling, including electrical conductivity data. The ability to calculate conductivity opens up a wide range of experimental data for comparison which can help to define ambiguous quantities such as the average ion charge and refine the model parameters, in particular the exchange and correlation potentials. Investigations into improvements to the LDA treatment of exchange and correlation potentials are ongoing.

\section{Acknowledgements}

This work was performed under the auspices of the U.S. Department of Energy by University of California Lawrence Livermore National Laboratory under contract No. W-7405-Eng-48. We are grateful to Alan DeSilva for sharing his electrical resistivity data and to Hugh DeWitt, Dima Yakovlev, and Alexander Potekhin for valuable discussions on the transport calculations. 


\section{References}

[1] B. Wilson, V. Sonnad, P. Sterne, and W. Isaacs, J. Quant. Spectrosc. Radiat. Transfer 99 (2006), 658.

[2] D.A. Liberman, Phys. Rev. B 20 (1979), 4981.

[3] F. Perrot, Phys. Rev. A 42 (1990), 4871 and Phys. Rev. E 47 (1993), 570.

[4] F.J. Rogers and D.A. Young, Phys. Rev. E 56 (1997), 5876.

[5] C. Blancard and G. Faussurier, Phys. Rev. E 69 (2004), 016409.

[6] J.C. Pain, G. Dejonghe and T. Blenski, J. Quant. Spectrosc. Radiat. Transfer 99 (2006), 451.

[7] L.H. Thomas, Proc. Camb. Phil. Soc. 23 (1927), 542.

[8] E. Fermi, Zeits. f. Phys. 48 (1928), 73.

[9] R.P. Feynman, N. Metropolis, and E. Teller, Phys. Rev. 75 (1949), 1561.

[10] R.M. More, K.H. Warren, D.A. Young and G.B. Zimmerman, Phys. Fluids 31 (1988), 3059.

[11] L. Hedin and B.I. Lundqvist, J. Phys. C 4 (1971), 2064.

[12] U. Gupta and A.K. Rajagopal, Phys. Rev. A 21 (1980), 2064.

[13] S.H. Glenzer, G. Gregori, R.W. Lee, F.J. Rogers, S.W. Pollaine, and O.L. Landen, Phys. Rev. Lett. 90 (2003), 175002.

[14] S.B. Hansen, W.A. Isaacs, P.A. Sterne, B.G. Wilson, V. Sonnad, and D.A. Young, Proceedings of NEDCP (2005), UCRL-PROC-218150.

[15] R. Evans, B.L. Gyorffy, N. Szabo, and J.M. Ziman, in The Properties of Liquid Metals, edited by S. Takeuchi (Wiley, New York, 1973).

[16] G.A. Rinker, Phys. Rev. B 31 (1985), 4207 and G.A. Rinker, Phys. Rev. A 37 (1988), 1284.

[17] F. Perrot and M.W.C. Dharma-wardana, Phys. Rev. A (1987) 36, 238 and F. Perrot and M.W.C. Dharma-wardana, International Journal of Thermophysics 20 (1999), 1299.

[18] J.K. Yuan, Y.S. Sun, and S.T. Zheng, Phys. Rev. E 53 (1996), 1059.

[19] D.A. Baiko, A.D. Kaminker, A.Y. Potekhin, and D.G. Yakovlev, Phys. Rev. Lett. 81 (1998), 5556.

[20] A.Y. Potekhin, D.A. Baiko, P. Haensel, and D.G. Yakovlev, Astronomy and Astrophysics 346 (1999), 345.

[21] W.R. Johnson, C. Guet, and G.F. Bertsch, J Quant. Spectrosc. Radiat. Transfer 99 (2006), 327. 
[22] A.W. DeSilva and J.D. Katsouros, Phys. Rev. E 57 (1998), 5945; ibid. 59, (1999) 3774; J.W. DeSilva and J.D. Katsouros, Proceedings of the International Conference on Strongly Coupled Coulomb Systems, St. Malo, France, (2000).

[23] K. Widmann, T. Ao, M.E. Foord, D.F. Price, A.D. Ellis, P.T. Springer, and A. Ng, Phys. Rev. Lett. 92 (2004), 125002. 

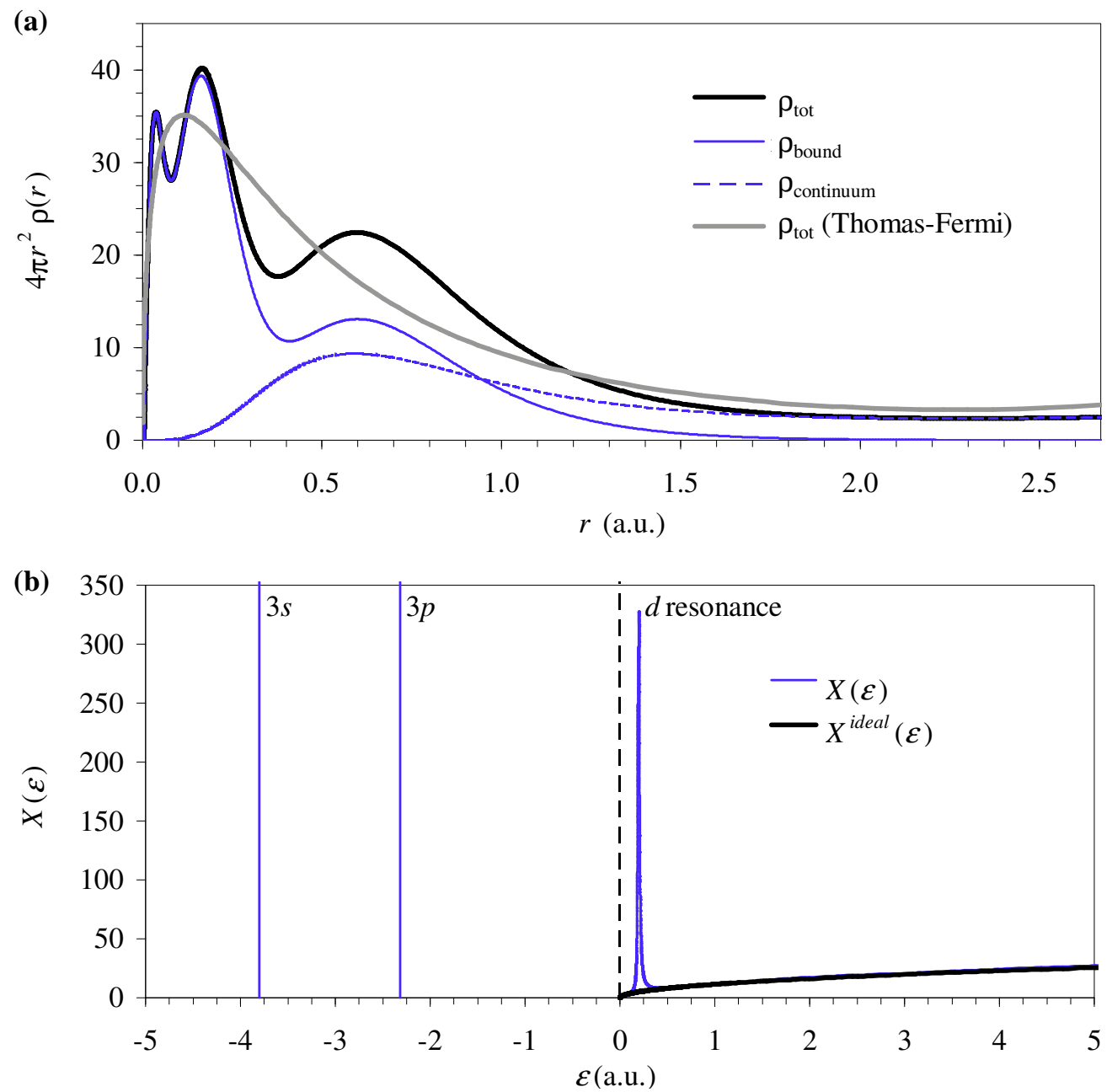

Fig. 1. Calculations for $\mathrm{Cu}$ at $1 \mathrm{eV}$ and solid density $\left(8.92 \mathrm{~g} / \mathrm{cm}^{3}\right)$. (a) Electron distributions within the Wigner-Seitz cell. The gray line is the distribution in the Thomas-Fermi approximation. (b) Bound state energy eigenvalues $(\epsilon<0)$ and continuum $X(\epsilon>0)$, which exhibits a resonance feature corresponding to the pressure-ionized $3 d$ orbital. 


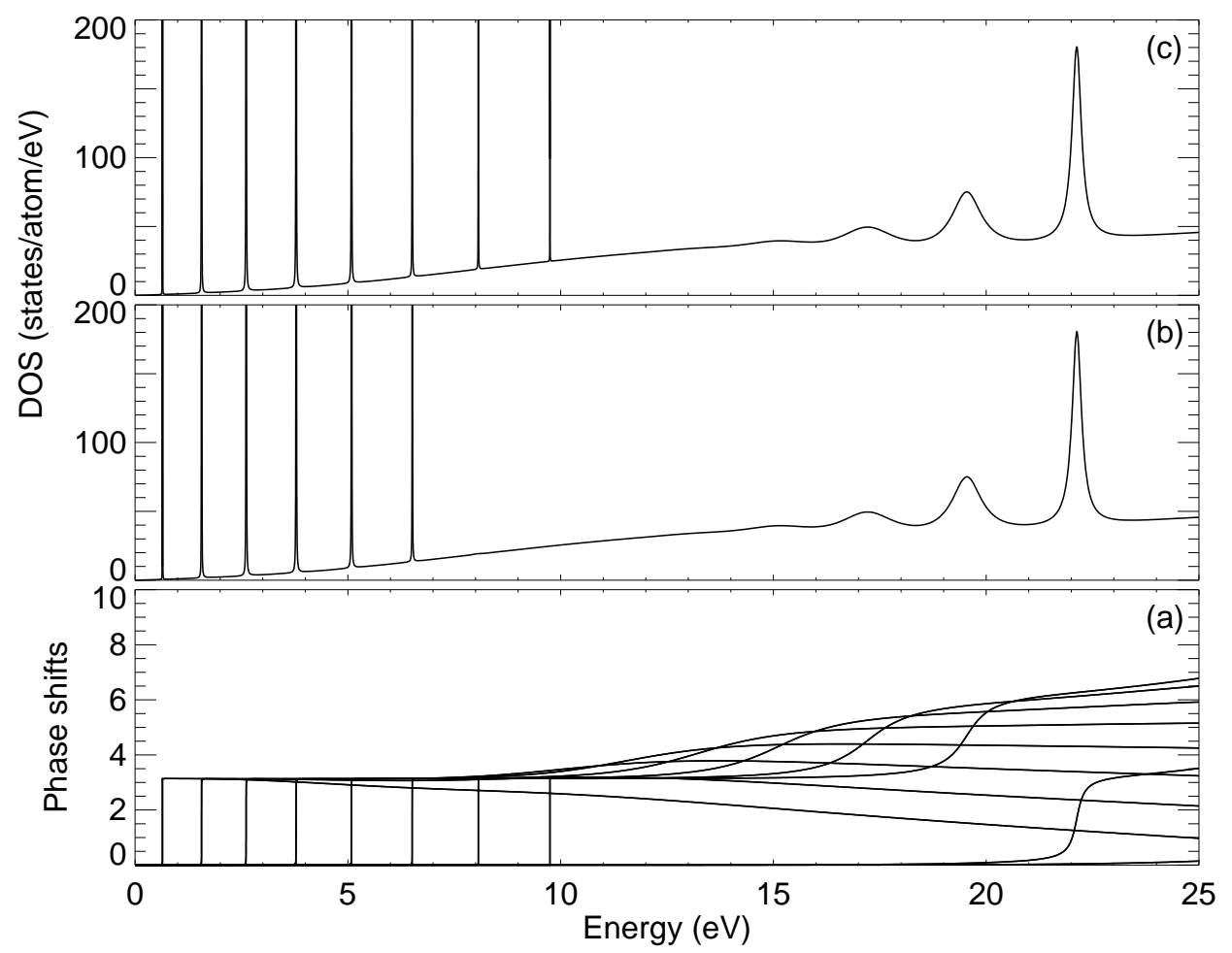

Fig. 2. Phase shifts and density of states for Mo at temperature $100 \mathrm{keV}$ and density $0.14 \mathrm{~g} / \mathrm{cc}$ (a) Phase shifts for partial waves with $|\kappa| \leq 15$. (b) DOS calculated using Gauss-quadrature-based adaptive energy grid only. Note missing peaks between 8 and $10 \mathrm{eV}$. (c) DOS calculated using phase-shift-tracking and adaptive energy grid. 


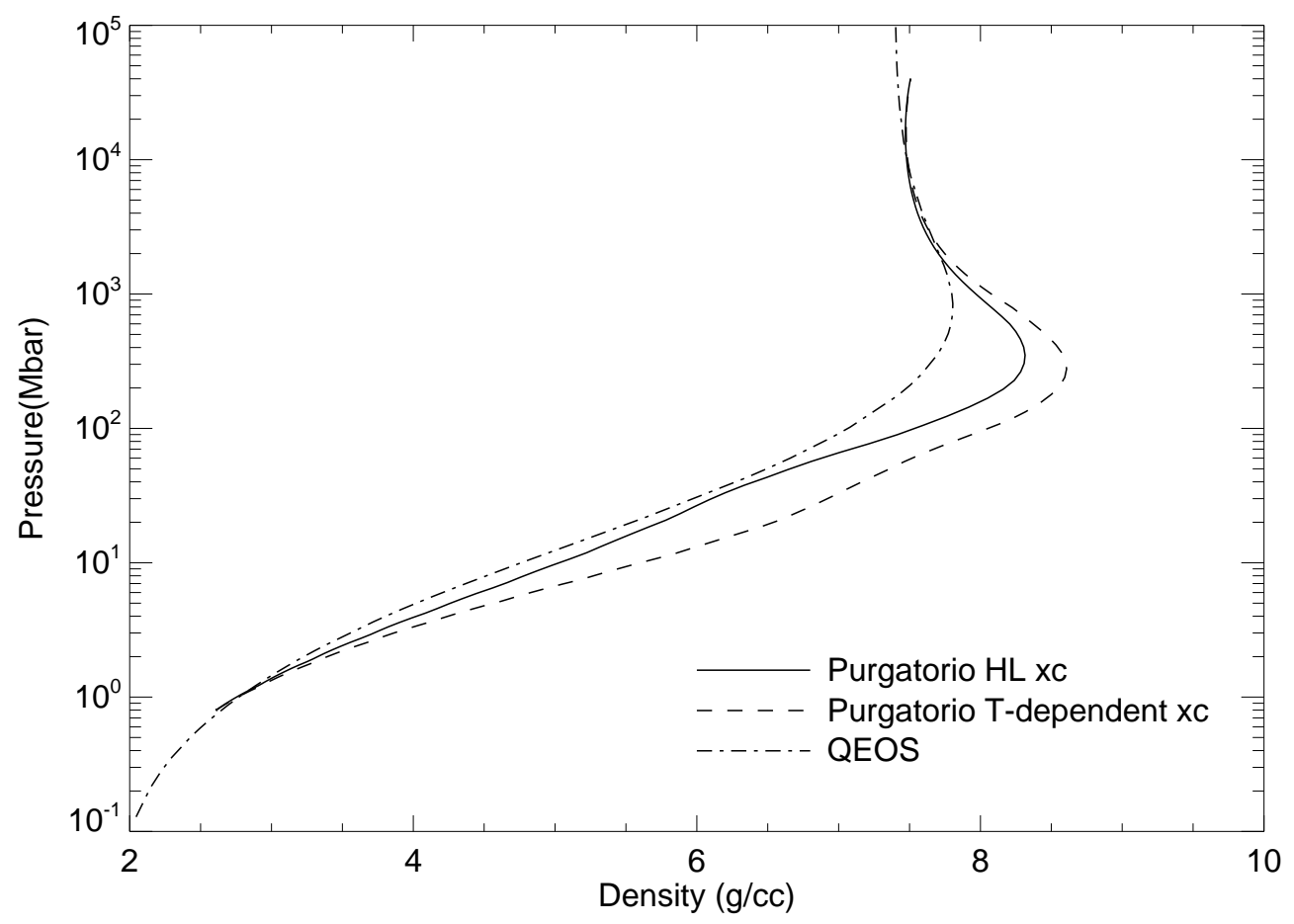

Fig. 3. Beryllium principal Hugoniots calculated with Hedin-Lundquist (HL) and temperature-dependent exchange potentials compared with the Thomas-Fermi-based QEOS model result. 


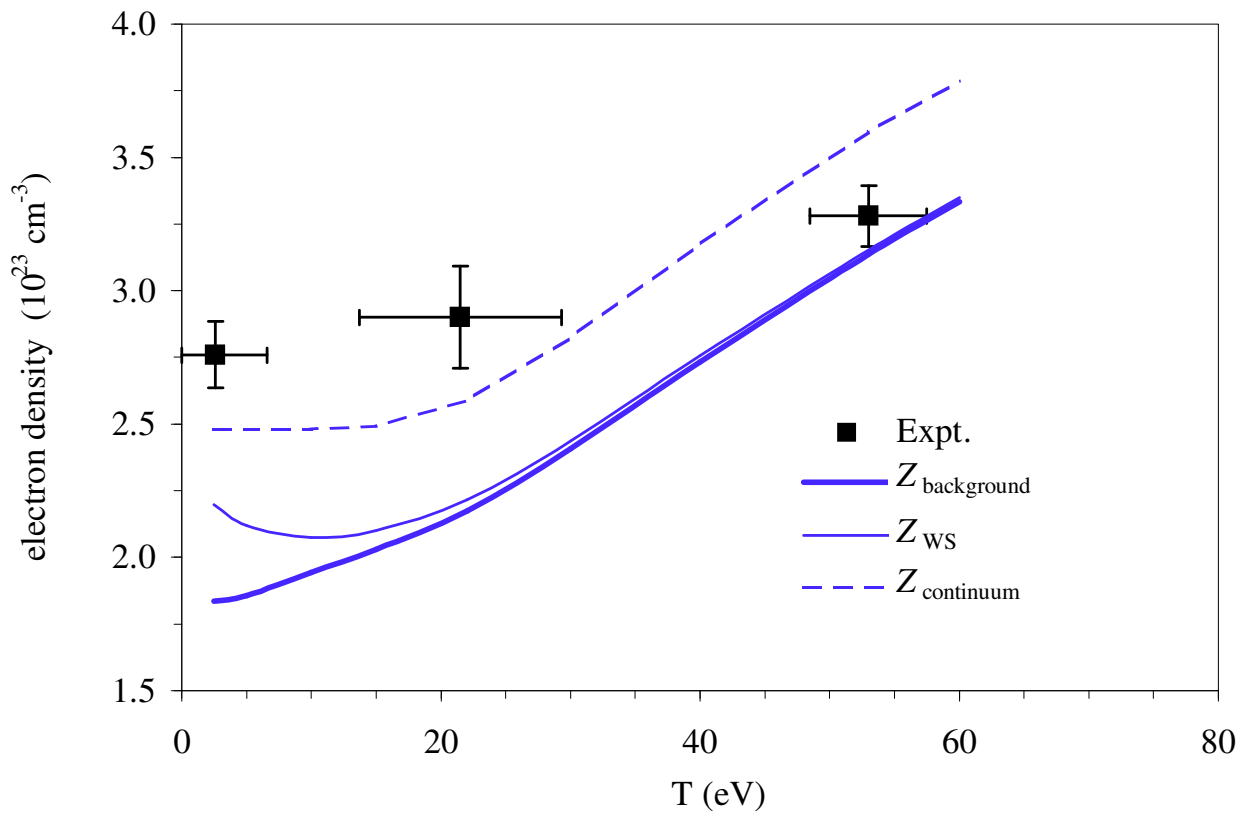

Fig. 4. Electron density of $1.85 \mathrm{~g} / \mathrm{cm}^{3}$ Be as measured by x-ray scattering [13] and calculated by Purgatorio with three different formulations of the average ion charge. 

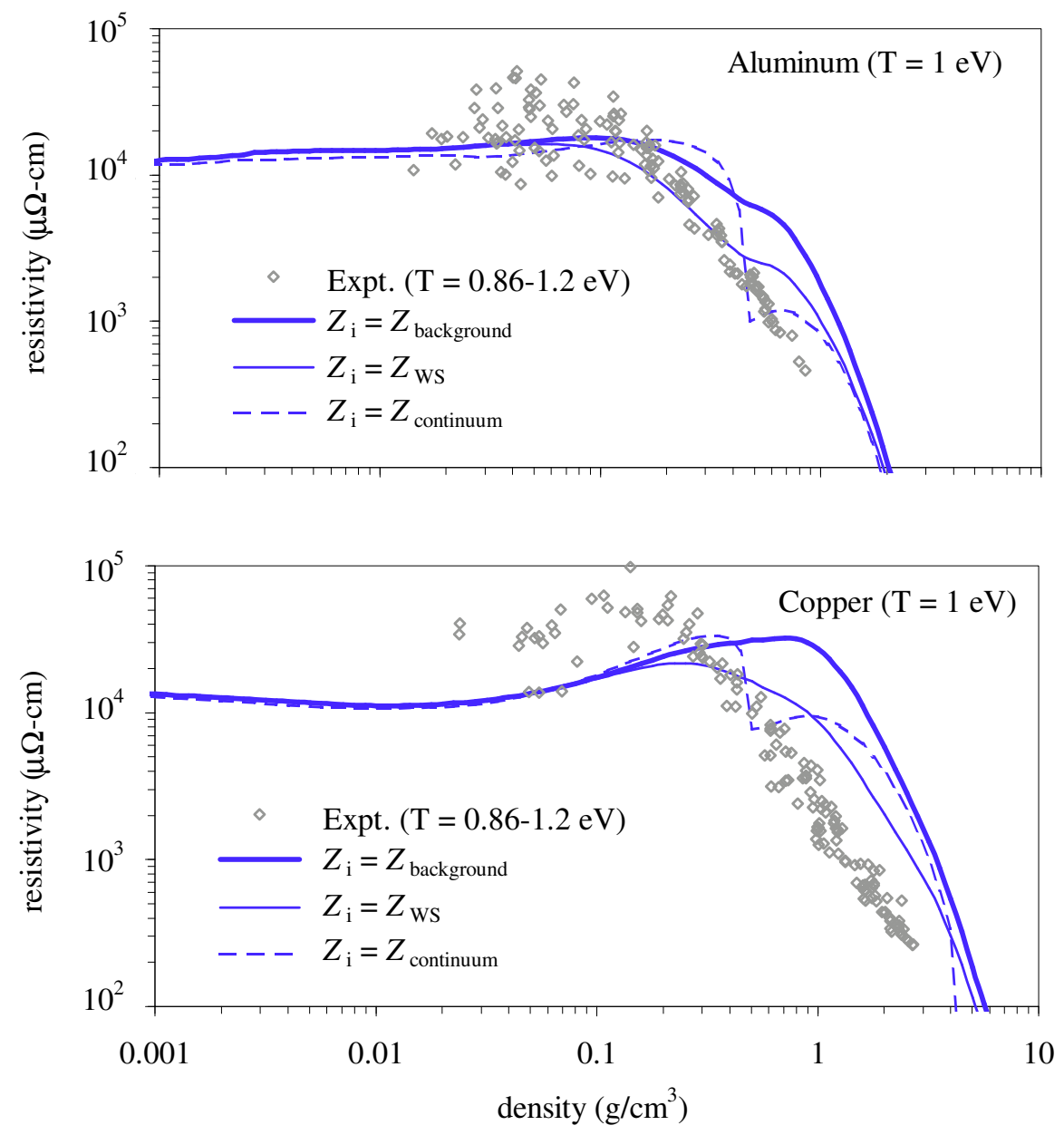

Fig. 5. Comparison of calculated electrical resistivities for $\mathrm{Al}$ and $\mathrm{Cu}$ as a function of material density showing the effect of different selections for the number of charge carriers $Z_{i}$. Data points are from Ref [22]. 